\title{
References to Research Literature in QA Forums - A Case Study of zbMATH Links from MathOverflow
}

\author{
Fabian Müller, Moritz Schubotz and Olaf Teschke (all FIZ Karlsruhe, Berlin, Germany)
}

Since August 2017, MathOverflow [1] users can seemlessly detect literature references in zbMATH [2] and insert them into discussions. Likewise, backlinks are automatically generated. This feature has been used in more than 500 distinct questions since then. In this column, we discuss the details of this tool and analyse its usage data during the last two years.

\section{Motivation}

Q\&A services like MathOverflow and review services like zbMATH are both driven by active communities. Although they address quite different needs of a working mathematician, there are many occasions where it is highly useful to have both information facets available. As a typical example, we may refer to our earlier column [3] on mathematical pseudonyms in zbMATH author profiles, which greatly benefited from a respective MathOverflow thread [4] (and, vice versa, we could add a lot of further answers to it derived from our author database). Another helpful thread was a discussion on the documentation of academic misconduct in review services [5]. It generated valuable feedback which helped to identify and document several cases of plagiarism.

The most natural motivation for interlinking both sources obviously stems from the need of supporting questions and answers in the platform with references to the literature, ideally with minimal barriers for users. Backlinks generated in this course would add interesting information beyond the reviews. As such, the realisation of such an interface would be very much in the spirit of building the envisioned Global Digital Mathematics Library of interlinked services [6].

\section{Linking MathOverflow and zbMATH}

In order to do so, we started a collaboration in 2016 with MathOverflow to set up an intuitive and easy-to-use interface for citing literature sources by harvesting the power of the zbMATH database.

For this, we were able to make use of the existing technology we use for various kinds of matching tasks, like finding the zbMATH document which a certain reference from a document refers to. This is a non-trivial task that uses modern methods of machine learning in connection with domain-specific indexing technologies. The case for MathOverflow matching is actually an easier one: when people search for a mathematical paper, they will usually enter one or more author names plus a couple of relevant terms from the title of the paper. It thus suffices to search for these terms in the author and title fields and return the most promising results. In a mechanical task without human intervention this wouldn't be quite enough to ensure a high level of accuracy, but since there is the additional step of the user selecting the paper they want from a short list of top hits, it is entirely enough for this type of use.

On the zbMATH side, we realised this functionality by implementing a small wrapper API around the existing matching functionality. We have done this in the past for other services as well, including e.g. ProjectEuclid [7] or EuDML [8].

On the MathOverflow side, Scott Morrison implemented a small front-end that can be reached via the newly made "Insert Citation" button (see Figure 1) when composing a new question or answer. The search results are displayed including preview links to the articles at zbMATH and, if available, in other places like arXiv or the publisher's website, so if the user is not entirely sure it is the article they want they can easily have a look and check. On confirmation the citation is inserted, correctly formatted, into the MathOverflow question or answer, including bibliographic information, a zbMATH link and a DOI link if available.

Finally, having the information on MathOverflow is helpful for the community there, but it is similarly interesting for the zbMATH users to discover that a certain article has been discussed on MathOverflow. A regularly running script collects these using the StackExchange API ${ }^{1}$ and displays them as backlinks together with the cited zbMATH articles. It thus contributes a small but

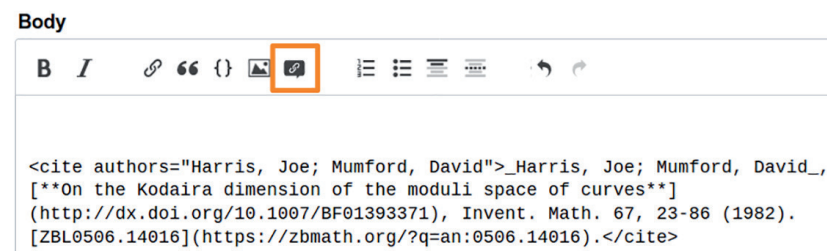

Harris, Joe; Mumford, David, On the Kodaira dimension of the moduli space of curves, Invent. Math. 67, 23-86 (1982). ZBL0506.14016.

Fig. 1. Inserting a citation on MathOverflow.

\footnotetext{
1 https://api.stackexchange.com/ (StackExchange is the network of Q\&A sites for various fields of interest of which MathOverflow is a part).
} 
helpful further step towards findability of mathematical ideas and interoperatibility of mathematical research tools. In the following, we investigate how this feature has been used in the past in Section 3 and discuss future perspectives in Section 4.

\section{Statistical Analysis of Established Links}

The tool described in Section 2 was quickly adopted by the community. With Fields medalist Terry Tao being an early adopter (see Figure 2), by now 223 registered user accounts at MathOverflow have used the feature. ${ }^{2}$ As outlined in Figure 3, the average usage has increased over time (though the rate increases at a small rate, also due to the fact that the initial usage after its introduction was already quite high). If one normalises the chart with regard to the overall number of posts, one sees a slight increase in the relative number of posts over the years (2017 (incubation year) 1.23\%, 2018 (full year) $1.45 \%, 2019$ (ongoing year) $1.34 \%$ ). Note that the MathOverflow users are most active in April and their activity drops to about $85 \%$ of the April activity in June and July.

I'm not sure if it was the first, but the bounds would follow from the main theorem in Nair, Mohan; Tenenbaum, Gl'erald, Short sums of certain arithmetic functions, Acta Math. 180, No.1, 119-144 (1998). ZBL0917.11048.

See also the more uniform bounds (in your case, this would give uniformity in $h$, after taking the singular series into account) obtained in

Henriot, Kevin, Nair-Tenenbaum bounds uniform with respect to the discriminant, Math. Proc. Camb. Philos. Soc. 152, No. 3, 405-424 (2012); erratum ibid. 157, No. 2, 375-377 (2014). ZBL1255.11048.

The result also follows from the properties of the pseudorandom majorants for multiplicative functions such as $d_{k}$ that were constructed by Matthiesen, see e.g. https://arxiv.org labs/1606.04482

share cite improve this answer

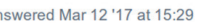
ก Terry Tao Ta.1k $20 \cdot 267 \cdot 354$

Fig. 2. Fields medalist Terry Tao uses the new feature [10].

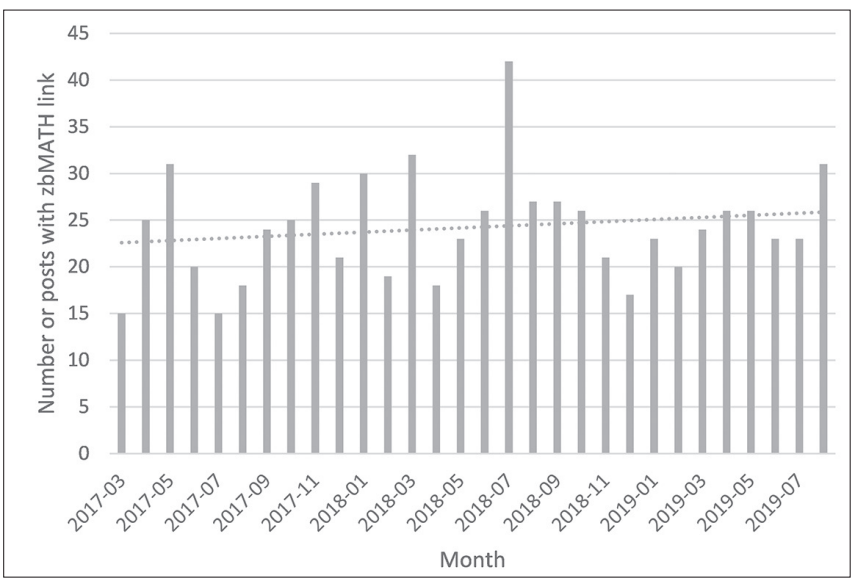

Fig. 3: Number of MathOverflow posts with a zbMATH link.

2 One may add that for almost as many users, their MathOverflow profile has been added as link to their zbMATH author profile, which is possible through the zbMATH community interface available at https://zbmath.org/author-profile/edit/ of from the author profile pages, cf. [11].

\section{Links between MathOverflow and zbMATH}

Overall 680 distinct zbMATH entries link to MathOverflow questions. The book $A$ primer on mapping class groups [9] is the entry with most links (4) to discussions on MathOverflow.

In the reverse direction, 512 questions link from MathOverflow to zbMATH. Of those links 16\% appear in the question and $83 \%$ in the answers. Here, one post [12] has 14 links to zbMATH.

\section{Subjects according to MSC}

The subjects of these posts (as reflected by the main area of the cited literature according to the Mathematical Subject Classification) reflect the most active communities in MathOverflow: algebraic and differential geometry; logic and category theory; manifolds and topology; and number and group theory account for more than half of these contributions.

Overall, we think that further investigation of the linking behaviour is of interest to the mathematical information retrieval community. Therefore, we provide the dataset together with a short usage guide on GitHub (see https://purl.org/zb/9).

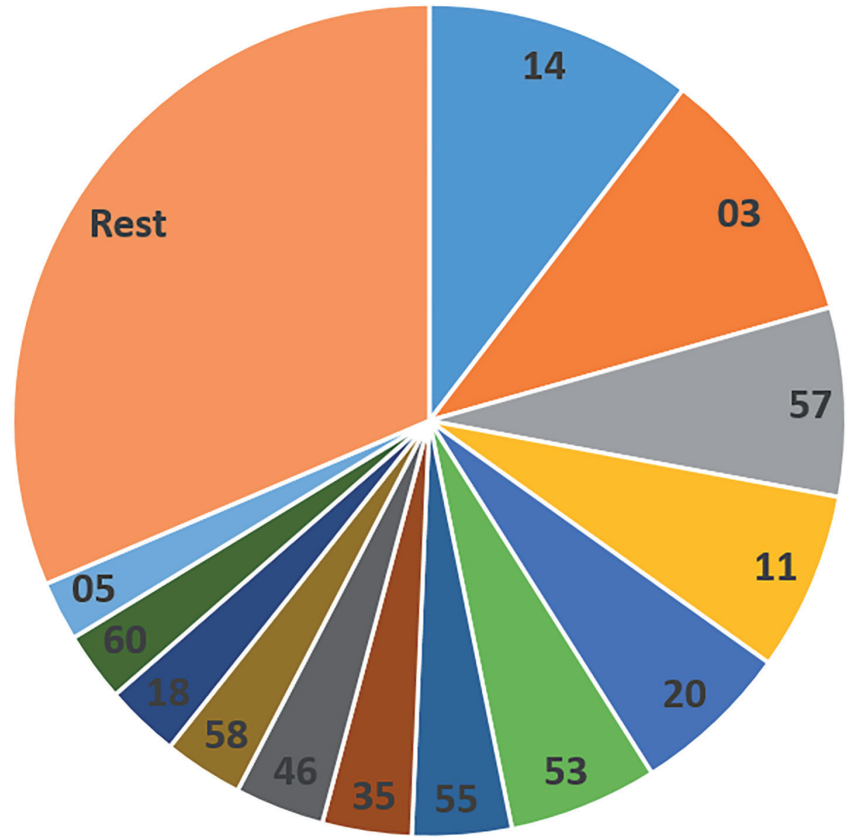

Fig. 4: Main MSC areas of zbMATH entries cited in MathOverflow.

\section{Conclusion and future work}

While linking academic literature from a question answering site such as MathOverflow was realised and adopted by the community, only $1 \%$ of the threads use that new feature. Questions in particular often do not reference academic literature. Here, advances in mathematical information retrieval technology might become helpful and suggest relevant academic literature related to the questions. While Corneli and Schubotz [13] envisioned that interlinking maths questions and Wikipedia articles might automatically resolve some questions, and a similar argument holds for MathOverflow questions and relevant academic literature. However, until now 
the machine understanding of mathematical questions is not yet developed enough to reasonably suggest relevant literature if the author or title of the publication are unknown to the author of the questions. Intelligent mathematical information retrieval technology and clever combinations of text and maths search engines might be able to suggest relevant literature in the future and thus help future question authors to resolve some questions by themselves.

\section{References}

[1] MathOverflow, https://mathoverflow.net/

[2] zbMATH, https://zbmath.org

[3] O. Paniagua Taboada, N. Roy and O. Teschke, Pseudonyms and author collectives in zbMATH. Eur. Math. Soc. Newsl. 109, 53-54 (2018; Zbl 1401.01047), https://zbmath.org/1401.01047

[4] D. Serre, "Pseudonyms of famous mathematicians", https://mathoverflow.net/questions/45185

[5] S. Kohl, "Frequency of papers showing academic misconduct among the articles indexed by MathSciNet and Zentralblatt MATH", https://mathoverflow.net/questions/267543 \#264442

[6] P. D. F. Ion and S. M. Watt, "The Global Digital Mathematics Library and the International Mathematical Knowledge Trust." Lect. Notes Comput. Sci. 10383, 56-69 (2017; Zbl 1367.68335)
[7] Project Euclid https://projecteuclid.org

[8] EuDML https://eudml.org

[9] B. Farb and D. Margalit, A primer on mapping class groups. Princeton, NJ: Princeton University Press (2011; Zbl 1245.57002), https:// zbmath.org/1401.01047

[10] K. Smith, "Who proved the upper bound for the autocorrelation of higher order divisor functions?", https://mathoverflow.net/questions/264436

[11] H. Mihaljević-Brandt and N. Roy, "zbMATH author profiles: open up for user participation”, Eur. Math. Soc. Newsl.93, 53-55 (2014; Zbl 1302.68304)

[12] Victor, "Classification of surfaces and the TOP, DIFF and PL categories for manifolds", https://mathoverflow.net/questions/96670

[13] J. Corneli and M. Schubotz, "math.wikipedia.org: A vision for a collaborative semi-formal, language independent math(s) encyclopedia," in 2nd Conference on Artificial Intelligence and Theorem Proving, Obergurgl, Austria, March 26-30, (2017; http://aitp-conference. org/2017/aitp17-proceedings.pdf)

Pictures and CVs of the authors can be found in previous Newsletter issues.

\section{Book Reviews}

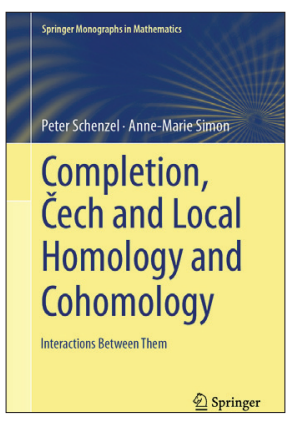

Peter Schenzel

Anne-Marie Simon

Completion, Čech and Local

Homology and Cohomology

Springer, 2019

$\mathrm{xvii,} 346 \mathrm{p}$.

ISBN 978-3-319-96516-1 ated ideals resp. ideals generated by weakly pro-regular sequences. A main technical tool is the Čech complex of a system of elements and its free resolution. In several results, the authors construct explicit morphisms that are quasi-isomorphisms between complexes describing isomorphisms in the derived category.

The book is divided into three parts. The first one is devoted to modules, the second part is devoted to the study of complexes and the part three is mainly concerned with duality.

In Chapter 1 the authors deal with preliminaries about complexes and also recall direct and inverse limits. Chapter 2 starts with basic results on adic completion. the authors study the left drived functors of the completion and then they study the class $\mathscr{C}_{\mathfrak{a}}$ of modules with the property that the left derived functors of the $a$ adic completion vanish in positive degree, while in degree aero it coincides with the a-completion functor. In Chapter 3, the authors give a generalization completeness criterion due to Jensen: "Let $(R, \mathfrak{m})$ be a Noetherian local ring. Then, a finitely generated $R$-module $M$ is complete in its $\mathrm{mt}$-adic topology if and only if $\operatorname{Ext}_{R}^{i}(F, M)=0$ for all $i>0$ and any flat $R$-module $F$ ". In Chapter 4 , they summarize the work of Avramov and Foxby on unbounded complexes. In addition, it is presented a study of minimal injective resolutions of unbounded complexes, including the case of not necessarily Notherian rings. Chap- 